\title{
Analysis of Chromosome Morphology and Possible Means of Speciation in Jasminum
}

\author{
Arun Kumar Sharma and Archana Sharma \\ Cytogenetics Laboratory, Department of Botany, Calcutta University, \\ 35, Ballygunge Circular Road, Calcutta 19, India
}

Received December 7, 1957

\section{Introduction}

The different species of Jasminum are very favourite plants of horticulturists, due chieffy to the lovely fragrant flowers borne by them. They are extensively cultivated in different nurseries and gardens throughout India,-a factor which is largely responsible for the origin of a number of horticultural varieties of this genus. The principal means of their propagation is through cuttings. Of all the species, J. sambac, because of its wide demand, is most favoured in cultivation.

A general cytological study of this genus has been carried out by certain workers (Bowden 1940, Krishnaswamy and Raman 1948, 1949, Dutt 1952 etc). Their observations reveal that all the species are characterised by a basic set of thirteen chromosomes. The existence of some polyploid types is also on record. In a majority of the species however, twenty-six chromosomes constitute the $2 n$ complement. Suggestions have been forwarded, emphasizing the role of both auto and allopolyploidy in the evolution of its species.

Unfortunately, however, data on the detailed karyotype, which is expected to throw light on the mechanism of speciation in such a uniform genus, is extremely lacking. The figures of somatic chromosomes, presented by previous authors (Raman 1955), which are claimed to represent their morphology, do no reveal clearly even the primary constriction regions. Evidently technical limitations had been mainly responsible for the failure in clarifying the constriction regions and other details of the karyotypes in Jasminum, as the complements of its species are characterised by having very small chromosomes. It is indeed difficult to analyse the karyotype in detail with the classical methods which were followed by previous authors.

A critical investigation of the karyotypes in Jasminum, where the majority of the species possess the same chromosome number, is very necessary. Leaving aside the earlier works on Lilium (Stewart 1940), Crepis (Babcock 1942) etc., lately considerable amount of data have accumulated showing that, in most of the plant genera having species with uniform chromosome number, the species can be differentiated on the basis of their karyotypes (Mookerjea 1955 a, b, 1956; Sharma and Bhattacharyya 1956; Sharma and Sarkar 
1956 ; Sharma and Ghosh 1954; Sharma and Datta 1957; Sharma and Bhattacharyya 1957; Sharma and Bhattacharjee 1957; Sharma and Sharma 1957), etc. Such minute karyotypic differences, which were not recorded previously, could only be brought out following a number of improved techniques devised in recent years (Sharma 1956).

The authors too found the earlier techniques to be of very little use in investigating the detailed chromosome morphology of this genus. This difficulty accounts for the fact why data on this aspect could not be obtained previously. The lack of any information on this aspect of the cytology of Jasminum is regarded as constituting a major lacuna in our knowledge, which must be bridged to have a clear insight into the process of evolution within this horticulturally important genus. In view of this necessity, therefore, the present work was undertaken. When the commonly used techniques of clarification of chromosome morphology failed in the case of Jasminum, trials were given using techniques which involved pretreatment with a number of chemicals worked out in the last few years. As a result of these trials, a technique has been standardized, effective enough to bring out clearly the details of the karyotypes in this genus, previously unrecorded. It is hoped that all the observations outlined in the following paper would serve, not only to indicate the role of structural alteration in speciation within Jasminum, but also to emphasize the possibility of the detection of specific and varietal differences on the basis of their karyotypes.

\section{Materials and methods}

For the present investigation fourteen varieties of common Indian jasmines; distributed under nine species, have been studied, A list of the types, which have been cytologically worked out here, is given below:-
1) Jasminum auriculatum Vahl.
8) J. sambac
Ait. var. "sultanee Joyee"
2) J. angustifolium Willd.
9) J. sambac
Ait. var.
3) J. calophyllum Wall.
10) J. sambac "motia"
Ait. var.
"khoya"
4) J. grandiflorum Linn. var.
11) J. sambac
Ait. var. rai alba
12) J. sambac japanese
5) J. pubescens Willd. var. alba
6) J. pubescens Willd. var.
13) J. autumnale rubescens
Ait. var. rai double
7) J. sambac Ait. var.,
14) J. arborescens

The plants were obtained from the local nurseries and their identifica- 
tion was confirmed from the Herbarium at the Indian Botanical Gardens at Sibpore, Calcutta. They are all cultivated extensively as common ornamental plants in many gardens. They were grown in earthernware pots in shady areas in the University College gardens. A suitable mixture of sand, sawdust and manure was used to secure healthy roots.

Certain difficulty was experienced in studying the chromosomes of roottips of jasmines, due to their small size and poor stainability. Fortunately, a method could be worked out which gave well-scattered metaphase plates. Root-tips were treated in saturated Aesculine solution in water for 45 minutes to an hour at a temperature of $10^{\circ}-15^{\circ} \mathrm{C}$. They were then fixed in acetic alcohol $(1: 1)$ mixture for a period of 45 minutes. Subsequently, the roottips were heated in a mixture of $2 \%$ aceto-orcein solution and normal $\mathrm{HCl}$ $(9: 1)$ for a few seconds. They were kept in the mixture for half-an-hour and then smeared in 1\% aceto-orcein solution. Preparations with perfectly scattered metaphase plates with constriction regions well clarified were thus obtained.

The preparations were made permanent by inverting the slides in $n$ butyl alcohol till the cover-glasses were detached and subsequent mounting in canada balsam.

Meiotic studies were made from smears in Nawashin's fluid and stained following Newton's crystal violet iodine technique. Premordanting in 1\% chromic acid for overnight was found to be necessary to obtain bright staining.

Observations were made in Zeiss Winkel microscope and the figures were drawn at a table magnification of $\times 2800$, using a Zeiss compensating eyepiece $\times 20$ and a 1.3 apochromatic objective with an aplanatic condenser of 1.3 N.A.

In the diagrams, the chromosomes bearing satellites or secondary constrictions have been drawn in outline.

\section{Observations}

The somatic chromosomes of jasmines range from medium-sized to short, as compared to those of other genera. Size difference amongst the chromosomes is present, there being a graded series from the longest to the shortest. The normal somatic number has always been found to be a multiple of thirteen, ranging from $2 n=26$ to $2 n=52$. The number of chromosomes bearing secondary constrictions varies from four in $J$. angustifolium to ten in $J$. sambac var. khoya.

Somatic nuclei, bearing chromosomes of a different number from the normal one have been recorded in some varieties in the same tissue where normal nuclei are also present. In these cases, the number, which occurs in the highest frequency of cells, has been taken to be the normal one for that particular variety. 
A detailed analysis of the karyotypes of the different varieties studied, shows the presence of certain distinct types of chromosomes, different combinations of which are present in different species and varieties. In all, ten types are observed. A general description of the types based on their relative size and positions of primary and secondary constrictions is given below :-

1) Type A - A comparatively long chromosome, with two constrictions, primary and secondary, located at nearly median positions, close to each other.

2) Type B - A comparatively long chromosome, with two constrictions, primary and secondary, one nearly median in position and the other located at nearly subterminal position at the distal end of the slightly longer arm.

3) Type $\mathrm{C}-\mathrm{A}$ comparatively long chromosome, with two constrictions, primary and secondary, one nearly median in position and the other located at nearly submedian to nearly subterminal position at the distal end of the slightly shorter arm.

4) Type D - A comparatively long chromosome, with two constrictions, primary and secondary, one nearly submedian in position and the other nearly submedian at the opposite distal end of the longer arm.

5) Type $\mathrm{E}$ - A comparatively long chromosome, grading to nearly medium-size in some species, with a nearly median to nearly submedian primary constriction and a small satellite at the distal end of the shorter arm.

6) Type F - A comparatively long chromosome, grading to nearly medium-size with a submedian to nearly submedian primary constriction and a small satellite at the distal end of the longer arm.

7) Type $\mathrm{G}$ - A comparatively long chromosome, grading to nearly medium-size, with a median primary constriction and a small satellite at the distal end of one of the arms.

8) Type $\mathrm{H}-\mathrm{A}$ comparatively long chromosome, grading to medium-sizewith a submedian to nearly sub-terminal primary constriction.

9) Type I - Medium-sized chromosomes, ranging from nearly long to nearly short, with median to nearly submedian primary constrictions.

10) Type J - Short chromosomes with median to nearly median primary constrictions.

The species and varieties studied contain different numbers of the above mentioned types in their complements.

The result of the karyotype study of the different species and varieties is outlined below :-

1) Jasminum auriculatum Vahl. $(2 n=26)$

The normal number of chromosomes, present in the somatic cells, is twenty-six. Six of them are found to bear secondary constrictions. Size range varies from $2 \cdot 3 \mu$-to $1 \cdot 1 \mu$.

The chromosome complement is seen to consist of, two pairs of $\mathrm{E}$, one 

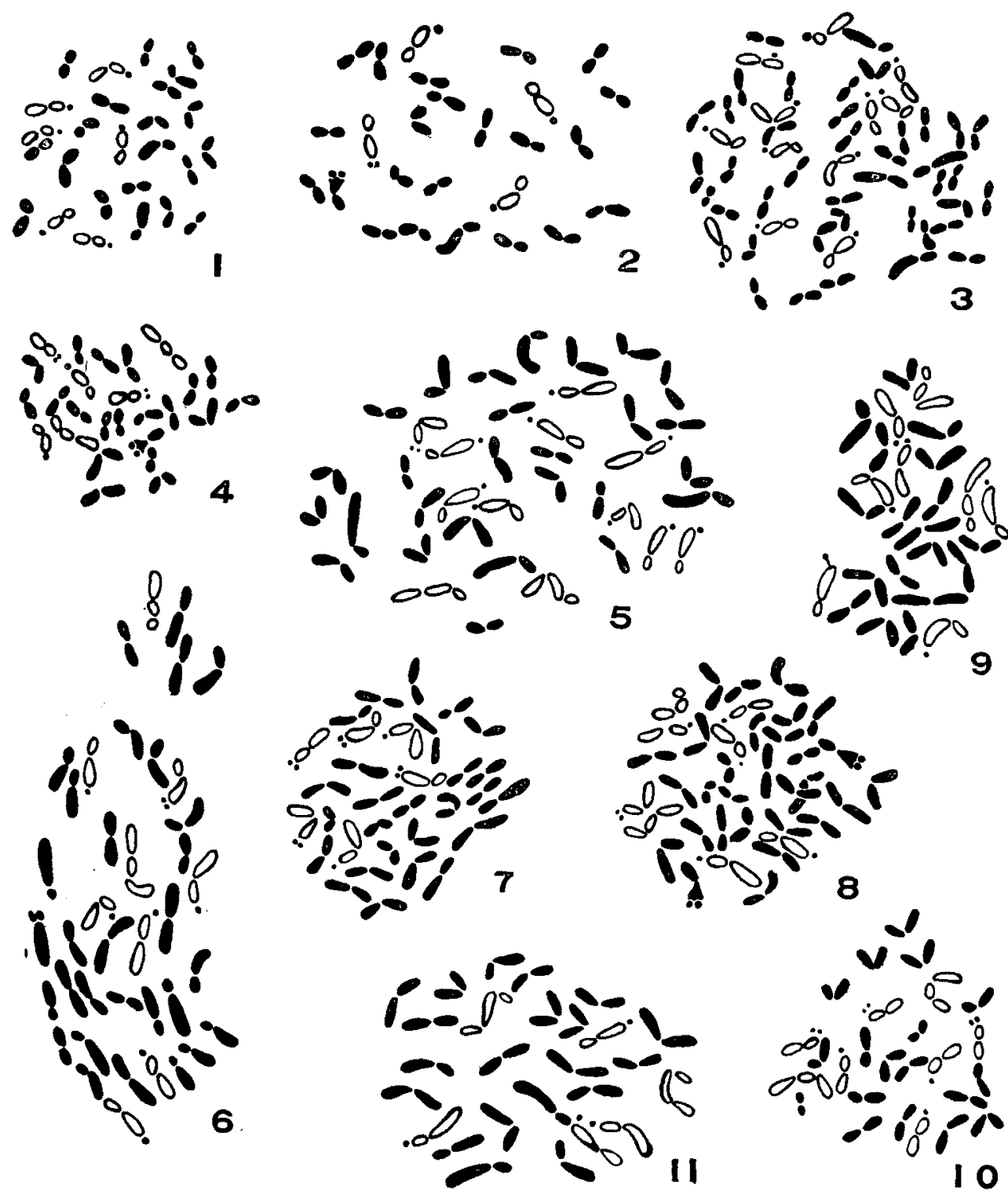

9
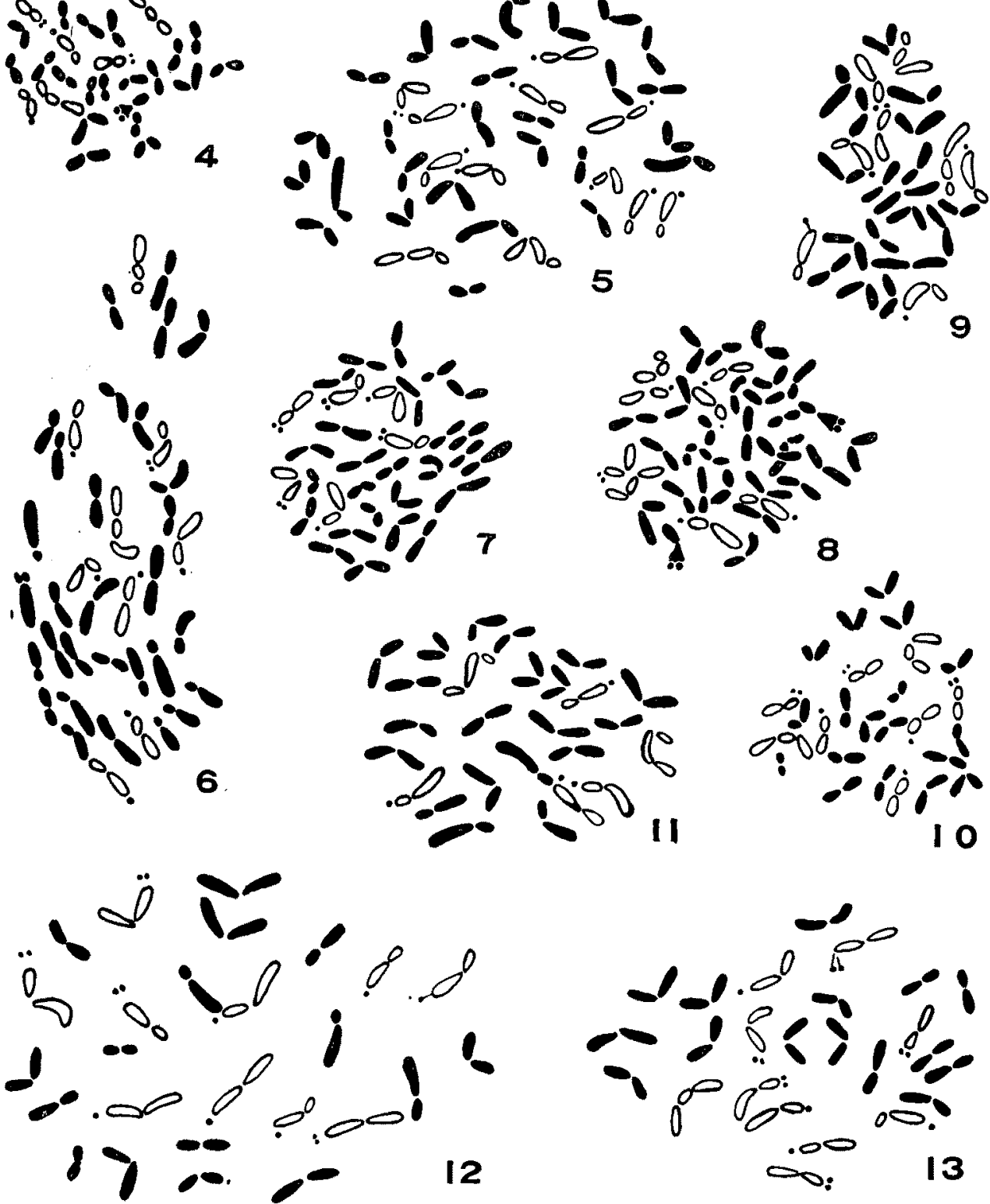

Figs. 1-13. 1-5, somatic metaphase plates of $J$. auriculatum $(2 n=26), J$. angustifolum $(2 n=26), J$. calophyllum $(2 n=52), J$. grandiflorum var. alba $(2 n=26)$ and $J$. pubescens var. alba $(2 n=39)$ respectively. $6-8, J$. pubescens var. mebescens $(2 n=39)$, normal somatic metaphase and variation nuclei with 32 and 37 chromosomes respectively. 9-11, somatic metaphase of $J$. sambac var. mallika $(2 n=26), J$. sambac var. sultanee jozee $(2 n=26)$ and $J$. sambac var. motia $(2 n=26)$ respectively. $12-13, J$. sambac var. khoua $(2 n=26)$. 
pair of $F$, two pairs of $\mathrm{H}$ and eight pairs of $\mathrm{I}$ type of chromosomes (Figs. 1 and 24, 1a). The I type chromosomes range from long to nearly short in size.

\section{2) J. angustifolium Willd. $(2 n=26)$}

The somatic complement is seen to be composed of twenty-six chromosomes. Four of them are found to bear satellities. The size ranges from $2 \cdot 5 \mu$ to $1 \cdot 1 \mu$.

In the complement, two pairs of $F$, one pair of $H$, nine pairs of $I$ and one pair of $\mathrm{J}$ type chromosomes are present. One $\mathrm{F}$ pair is slightly longer than the other (Figs. 2 and 24, 2a).

3) J. calophyllum Wall. $(2 n=52)$

In the somatic nuclei of this species, fifty-two chromosomes have been recorded. Twelve chromosomes are seen to bear secondary constrictions. The size range varies from $2.85 \mu$ to $1.1 \mu$.

A study of the karyotype shows the presence of the following types of chromosomes in the complement (Figs. 3 and 24,3a) - two pairs of $\mathrm{E}$, one pair of $G$, three pairs of $F$, two pairs of $H$, fourteen pairs of $I$ and four pairs of $J$ types. The $G$ chromosomes are longer than the $E$ and $F$ and the I type grades into the $\mathrm{J}$ type.

4) J. grandiflorum Linn. var. alba $(2 n=26)$

Twenty-six chromosomes are present in the normal somatic complement of the species. Six of them bear secondary constrictions. Size range varies between $2 \cdot 8 \mu$ and $1 \mu$.

The complement consists of - one pair of $A$, one pair of $F$, one pair of $\mathrm{E}$, one pair of $\mathrm{H}$ and nine pairs of I type chromosomes (Figs. 4 and 24, 4a). The $H$ and $F$ type chromosomes are longer than the $E$ type in this species.

5) J. pubescens Willd. var. alba $(2 n=39)$

Thirty-nine chromosomes have been recorded in the somatic cells of this species. Twelve of them bear secondary constrictions. The size ranges between $3 \cdot 2 \mu$ and $1 \cdot 2 \mu$.

The following types of chromosomes can be observed in the complement (Figs. 5 and 24, 5a):- one pair of $\mathrm{B}$ type, two pairs of $\mathrm{E}$ type, three pairs of $\mathrm{F}$ type, three individuals of $\mathrm{H}$ type, nine pairs of $\mathrm{I}$ type and three pairs of $\mathrm{J}$ type chromosomes. One pair of each of $\mathrm{E}$ and $\mathrm{F}$ type chromosomes are smaller than the other members belonging to the same type.

6) J. pubescens Willd. var. rubescens $(2 n=39)$

The normal somatic nuclei of the species are found to contain thirtynine chromosomes. Of them, nine bear secondary constrictions. The size ranges from $3 \cdot 9 \mu$ to $1 \cdot 5 \mu$.

A detailed study of the karyotype shows the presence of, one single chromosome of $\mathrm{A}$ and another of $\mathrm{C}$, two pairs of $\mathrm{E}$, three individuals of 
$\mathrm{F}$, two pairs of $\mathrm{H}$ and thirteen pairs of $\mathrm{I}$ type of chromosomes. One $\mathrm{F}$ chromosome is much smaller than its allies (Figs. 6 and 24,6a).

In addition to the normal karyotype described above, somatic nuclei
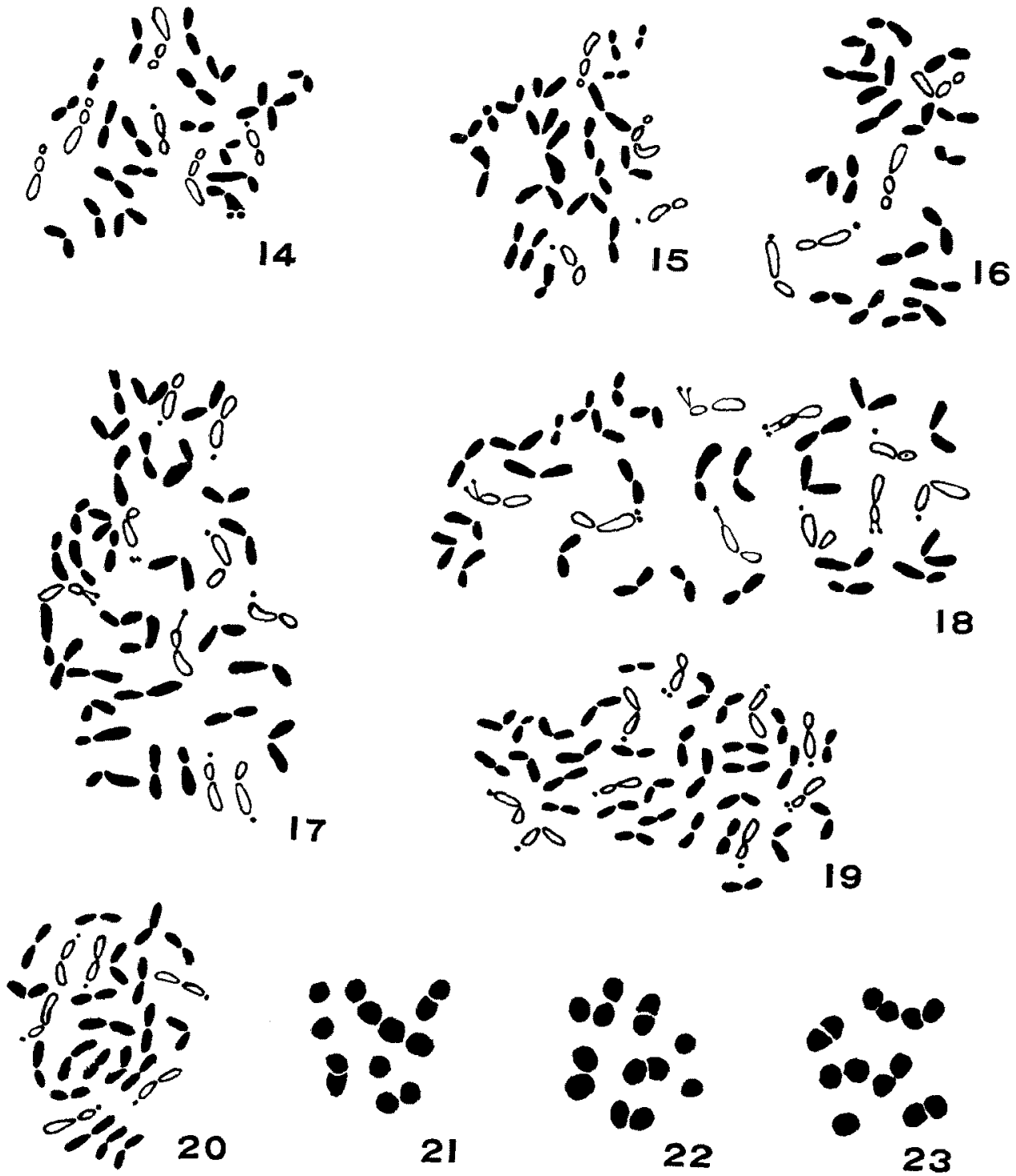

Figs. 14-23. 14-16, J. sambac var. rai japanese $(2 n=36)$, normal somatic metaphase and variation nuclei with 24 and 22 chromosomes respectively. 17-18, J. sambac var. rai double $(2 n=39)$, normal somatic metaphase and nuclei with 25 chromosomes respectively. 19-20, somatic metaphase of $J$. autumnale $(2 n=39)$ and $J$. arborescens $(2 n=26)$ respectively, 21-23, meiotic metaphase I of $J$. sambac var. mallika showing secondary association of bivalents.

with thirty-two (Fig. 7) and thirty-seven (Fig. 8) chromosomes are also on record. These sets have possibly arisen from the normal one through nondisjunction of chromosomes. 
7) J. sambac Ait var. " mallika" $(2 n=26)$

The somatic nuclei of this species contain twenty-six chromosomes. Of them, eight are seen to bear secondary constrictions. The size range varies from $3.9 \mu$ to $1.5 \mu$.

The complement consists of a pair of $\mathrm{C}$, a pair of $\mathrm{E}$, two pairs of $\mathrm{F}$ and nine pairs of I type of chromosomes. The $\mathrm{C}$ type and I type chromosomes are longer than those found in other species (Figs. 9 and 24, 9a).

8) J. sambac Ait. var. "Sultanee Joyee" $(2 n=26)$

Twenty-six chromosomes are present in the normal somatic complement of the species. Six of them are seen to bear secondary constrictions. The size range varies between $3 \cdot 2 \mu$ to $1 \cdot 1 \mu$.

The complement consists of a pair of $A$, a pair of $G$, a pair of $E$, nine pairs of $\mathrm{I}$ and a pair of $\mathrm{J}$ type chromosomes. The $\mathrm{G}$ type is longer than $\mathrm{E}$ in this species (Figs. 10 and 24, 10a).

9) J. sambac Ait. var. " motia" $(2 n=26)$

The somatic nuclei show twenty-six chromosomes in their complements. Six of them are seen to bear secondary constrictions. The size range varies between $4 \mu$ to $1 \cdot 9 \mu$.

The complement consists of a pair of $\mathrm{D}$, a pair of $\mathrm{E}$, a pair of $\mathrm{F}$, a pair of $\mathrm{H}$ and nine pairs of I type chromosomes (Figs. 11 and 24, 11a).

10) J. sambac Ait. var. "khoya" $(2 n=26)$.

Twenty-six chromosomes are present in the normal somatic complement of this species. In general, they are longer than those of the other species. Of these, ten chromosomes bear secondary constrictions. The size ranges between $4 \cdot 8 \mu$ to $1 \cdot 8 \mu$.

A detailed study of the karyotype shows the presence of the following types of chromosomes, a pair of $E$, two pairs of $G$, two pairs of $F$, seven pairs of $\mathrm{I}$ and one pair of $\mathrm{J}$ type of chromosomes. The $\mathrm{G}$ chromosomes are longer than the $\mathrm{E}$ type (Figs. 12 and 24, 12a).

In addition to the normal complement described above, a somatic nucleus with twenty-five chromosomes has also been recorded (Fig. 13). This set differs from the normal one in having an extra A type chromosome and in the absence of one $\mathrm{F}$ and one $\mathrm{I}$ type chromosomes. It has probably been derived from the normal set through non-disjunction and structural alterations.

\section{1) J. sambac Ait. var. rai japanese $(2 n=26)$}

The somatic nuclei show twenty-six chromosomes in their normal complement. Of these, six bear secondary constrictions. The size difference varies from $3 \cdot 2 \mu$ to $1 \cdot 1 \mu$.

The complement includes two pairs of $C$, one pair of $F$, a pair of $H$, six pairs of $\mathrm{I}$ and three pairs of $\mathrm{J}$ type of chromosomes (Figs. 14 and 24, 14a). 
In addition to the complement described above, cells with twenty-four and twenty-two chromosomes in their somatic sets have also been recorded (Figs. 15 and 16). These sets have possibly arisen from the normal one through non-disjunction.

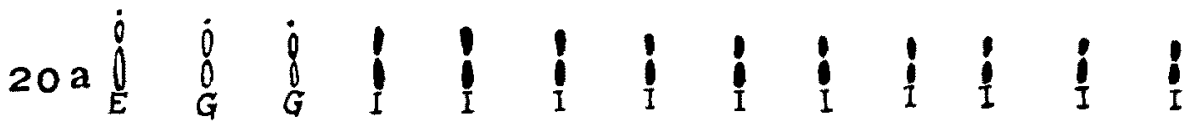

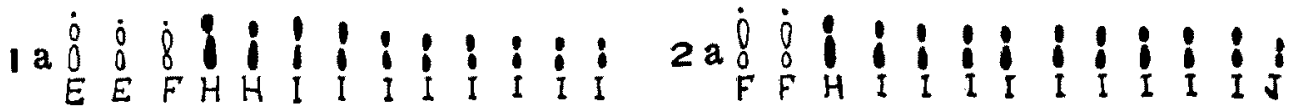
उаO巳

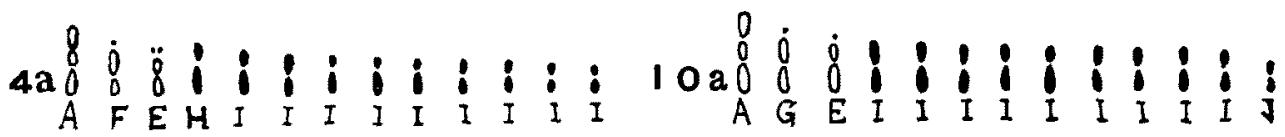

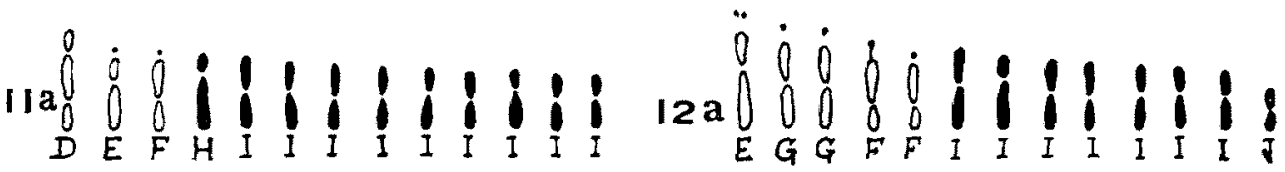

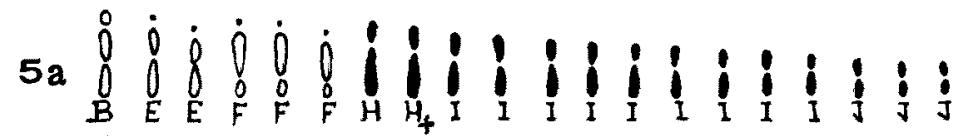

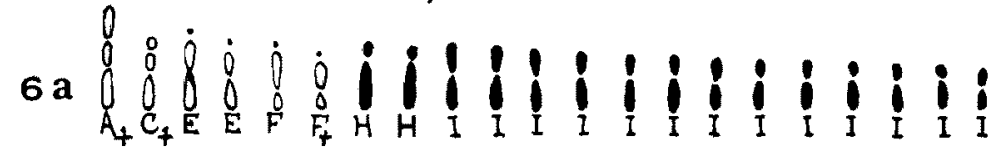

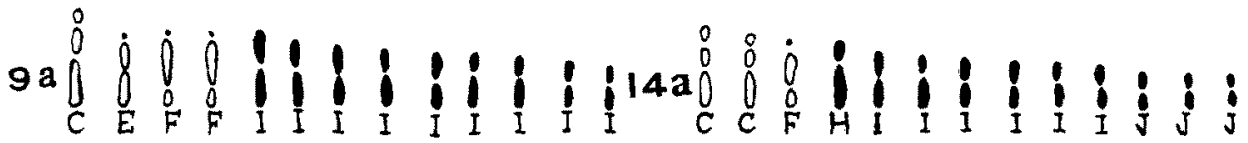

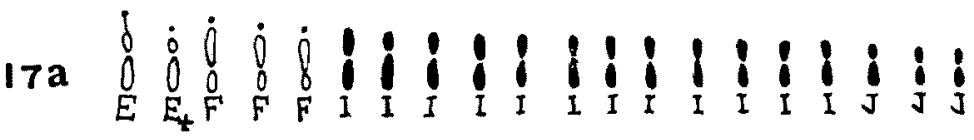

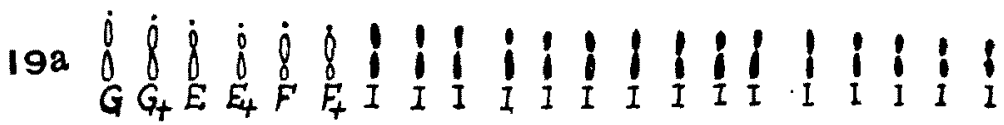

Fig. 24. Idiograms of Jasminum. The types marked with a t-denote that only an in. dividual member is present and not a pair. 1a, J. auriculatum. 2a, J. angustifolizum. 3a, J. calophyllum. 4a, J. grandiflorum var. alba. 5a, J. pubescens var. alba. 6a, J. pubescens var. mubescens. 9a, J. sambac var. mallika. 10a. J. sambac var. sultanee joyee. 11a, J. sambac var. motia. 12a, J. sambac var. khoya. 14a, J. sambac var. rai japanese. 17a, J. sambac var, rai double. 19a, J. autumnale. 20a, J. arborescens.

12) J. sambac Ait. var. rai double $(2 n=39)$

Thirty-nine chromosomes are present in the normal somatic complement. 
Of them, nine bear secondary constrictions. The size range varies from $3 \mu$ to $1 \cdot 2 \mu$.

In the karyotype, the following types of chromosomes are observed,three individuals of $E$ type, three pairs of $F$, twelve pairs of $I$ and three pairs of $\mathrm{J}$ type of chromosomes (Figs. 17 and 24, 17a).

In addition to the complement described above, variant nuclei with thirty-five chromosomes are also on record. Possibly this set has arisen from the normal one through non-disjunction (Fig. 18).

13) J. autumnale $(2 n=39)$

Thirty-nine chromosomes have been recorded in the somatic nuclei of this species. Of them, nine bear secondary constrictions. The size ranges from $2 \cdot 7 \mu$ to $1 \cdot 2 \mu$.

The complement includes, - three individuals of $G$ type, three individuals of $E$, three individuals of $F$ and fifteen pairs of $I$ type chromosomes (Figs. 19 and 24, 19a). The $G$ chromosomes are longer than $E$ and $F$.

\section{4) $J$. arborescens $(2 n=26)$}

The somatic nuclei contain twenty-six chromosomes in their complement. Six of them bear secondary constrictions. The size ranges between $3 \cdot 2 \mu$ and $1 \cdot 5 \mu$.

The complement includes, - a pair of $E$, two pairs of $G$ and ten pairs of I type chromosomes (Fig. 20 and 24, 20a).

\section{Discussion}

1) Difference in karyotypes and its implication in speciation

A review of the karyotypes presented above, of all the species and varieties of Jasminum studied, show that the karyotype is characteristic, not only for a species but also for each variety. In general, however, all the species show a superficial resemblance in their chromosome morphology in that all possess complements, the members of which do not show much size difference. The chromosomes bear median to submedian primary constrictions. The secondary constrictions and satellites come out clearly after pretreatment. The total amounts of chromatin matter of the different species, (leaving aside the polyploid ones), do not show much difference (vide Histogram). These similarities fully justify the inclusion of all of them under one common genus.

In spite of this resemblance in general features, the different species and varieties differ with respect of minute details of their karyotypes. From the observations, altogether ten general morphologically distinguishable types of chromosomes have been clearly recognized in their complements. These ten different types are present in varying proportions in the different species and varieties. Of these, a few types are rather uncommon and present in only 2 or 3 cases. For example, the type A with primary and secondary 
constrictions located close to each other at nearly median position, is present in only $J$. grandiflorum var. alba, $J$. pubescens var. rubescens and $J$. sambac var. sultanee joyee. Similar is the case with a number of other

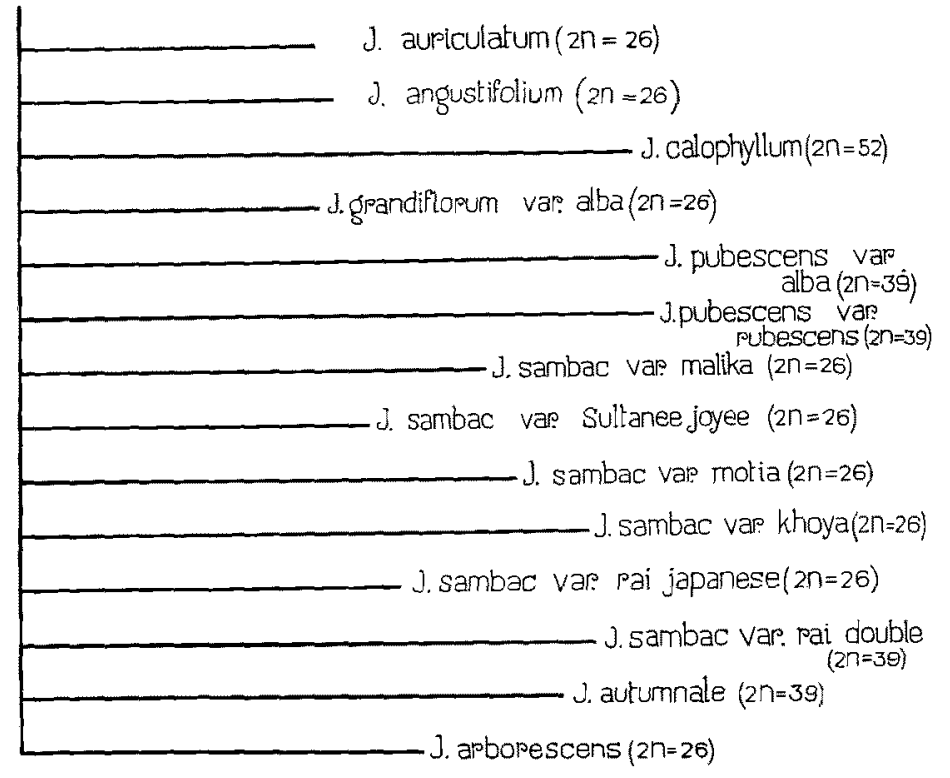

Fig. 25. Histogram of the types of Jasminum studied. The total amount of chromatin matter has been reduced to half in the diagram. types too. The number of satel. lites and secondary constrictions has been found to vary from four in $J$. angustifolium to ten in $J$. sambac var. khoya. Previously, only possibly approximate numbers of satellite and secondary constriction were suggested because no data could be obtained from metaphase chromosomes and observations were mainly based on the probable attachment of chromosomes in the prophase nucleus.

These structural differences of chromosomes between different species and varieties clearly indicate that speciation in this genus has been principally effected by structural alteration of chromosomes. The continued accumulation of structural changes has possibly been the main contributor to the origin of new species. The meiotic behaviour, which shows distinct bivalent formation, suggests that homozygosity for these changes has been attained by them. It is unfortunate that sexual reproduction is ineffective in most of the modern cultivated jasmines. Otherwises crossing between different varieties and species would have resulted in hybrids, the meiotic behaviour of which would have provided further evidences of the role of structural alteration in speciation.

2) Role of polyploidy in evolution

In addition to $2 n=26$ chromosomes in most of the jasmines studied, 52 and 39 chromosomes have been observed in $J$. calophyllum, and in two varieties of $J$. pubescens and one of $J$. sambac respectively. This data confirms the previous reports of the occurrence of some polyploid species within this genus. Meiotic behaviour of the polyploid species has been found to be regular, showing their allotetraploid nature, as suggested by previous 
authors (Raman 1955, Krishnaswamy and Raman l.c., etc). Meiotic observations have not been mentioned in the text as they confirm mainly data gathered by previous workers. Further evidence of their allotetraploid constitution is also indicated by the tendency of some of the bivalents to associate in groups (Figs. 21-23), as observed in $J$ sambac var. mallika. Allotetraploidy must have been attained at a period while the species still retained its capacity for sexual reproduction.

It is evident that in addition to the principal means of speciation, that is, chromosomal alterations in structure, polyploidy too has assisted to some extent initially in the speciation of this genus. But the duplication of the chromosome set should not be considered as the main factor responsible for the evolution of species, particularly in view of the fact that most of the species contain $2 n=26$ chromosomes.

It appears that the loss of an effective means of sexual reproduction and fertility is in no way related to the attainment of triploidy in some cases (e.g., J. sambac var. rai double $2 n=39$ ), because most of the diploid species too are sexually sterile. This sterility is therefore due either to gene mutations or structural changes of chromosomes, occurring at a certain level of evolution.

\section{3) Means of species origin}

It appears problematic as to how species and varieties, which are being continually raised in horticulture, originate in those plants, where sexual reproduction is absolutely ineffective except in a few cases. In such cases, even when seeds are occasionally formed, the principal means of propagation is through cuttings.

The solution to this problem seems to lie in the behaviour of chromosomes noted in the somatic tissue. It may be recollected that in a number of cases, mentioned in the text, varying types of complement, differing mostly in structure and also in the number of chromosomes in some cases, have been found in the same somatic tissue. Amongst such varying types of nuclei, the one representing the normal complement occurs in the highest frequency.

This peculiar behaviour, taken in conjunction with the asexual means of reproduction prevalent in the genus Jasminum, clearly indicates that possibly such somatic mutations help in speciation through their participation in the formation of daughter shoots. This type of chromosome behaviour has recently been recorded in a number of plant genera where reproduction is chiefly through vegetative means and its significance in speciation has also been pointed out (Sharma and Bal, 1956; Sharma 1956; Sharma and Majumdar 1955; Sharma and Sharma 1956; Mookerjea 1955, a and b).

It has already been emphasized that the species and varieties of this genus mainly differ with respect to minute details of their karyotypes. Such 
chromosomal changes possibly originate in the somatic tissue as shown by the presence of varying complements mentioned in the text. Their participation in the formation of daughter shoots and gradually of new individuals can only account for such karyotypic differences between varieties and species, where sexual reproduction is obsolete. This is a much more effective means of speciation for them as compared to means depending on the complex mechanism for successful fertilization.

\section{Summary}

A thorough study of the karyotypes has been performed on fourteen different species and varieties of Jasminum in order to fill this lacuna in our knowledge of the cytology of this genus. Improved techniques, involving the use of Aesculine, have been standardized for this purpose. The absence of any detailed study on this aspect has been attributed mainly to the limitations of the methods adopted previously.

A general resemblance has been noted in the chromosome morphology between species of Jasminum, indicating that the genus represents a natural assemblage. The different species and varieties have been found to differ from each other in minute details of the karyotype. This fact has been regarded to suggest the important role played by structural alteration of chromosomes in evolution. As each and every species or variety is characterized by a distinct karyotype, the significance of this study in the identification of species has been emphasized.

The minor part played by polyploidy and specially, allotetraploidy, before the species concerned lost its capacity for sexual reproduction, has also been pointed out.

Variation in chromosome complements has been noted in the same somatic tissue containing normal nuclei and, as sexual propagation is mostly ineffective in this genus, the role of such somatic mutations in speciation of this genus through asexual means has been suggested.

\section{References}

Babcock, E. B. 1942. Systematics, cytogenetics and evolution in Crepis. Bot. Rev. 8: 139190.

Bowden, W. M. 1940. Diploidy, polyploidy and winter hardiness relationships in the flowering plants. Amer. J. Bot. 27: 357-371.

Dutt, Mridula 1952. Chromosome numbers in some ornamental jasmines. Sci. Cult. 17: $527-528$.

Krishnaswamy, N. and Raman, V. S. 1948. Cytogenetical studies of the Indian Jasmines. I. Taxonomy and chromosome numbers of four varieties of $J$. sambac and other species J. Ind. Bot. Soc. 27: 77-83.

- and - 1949. A note on the chromosome numbers of some economic plants of India. Curr. Sci. 18: 375-378. 
Mookerjea, Archana 1955a. Cytology of Amaryllids as an aid to the understanding of evolution. Caryologia 7: 1-71.

- 1955b. Cytology of different species of Aroids with a view to trace the basis of their evolution. Caryologia 7:221-291.

- 1956. A cytological study of several members of the Liliaceae and their interrelationships. Ann. Bot. Soc. Vanamo 29: 1-44.

Raman, V. S. 1955. Cytogenetics of the Indian Jasmines II. The somatic chromosomes. Cytologia 20: 19-31.

Sharma, A. K. 1956. A new concept of a means of speciation in plants. Caryologia 9: 93-130.

- and Bal, A. K. 1956. A cytological study of a few genera of Amaryllidaceae with a view to find out the basis of their phylogeny. Cytologia 21:329-352.

- and Dipti Bhattacharjee 1957. Cytology of Sorghum I. Cytologia (in press). Abst. published in Proc. 44th Ind. Sci. Cong. 257, 1957.

- and Bhattacharyya, N. K. 1956. An investigation on the karyotype of the genus Cri um and its phylogeny. Genetica 28: 263-296.

- and Bhattacharyya, U.C. 1957. Cytological studies in Begonia I. La Cellule 58: 307329. Abst, published in Proc. 44th Ind. Sci. Cong. 259, 1957.

- and Dutta, P. 1957. Cytological investigation on the genus Ipomoea (in press). Abst. published in Proc. 44th Ind. Sci. Cong. 248, 1957.

- and Ghosh Chitra, 1954. Further investigations on the cytology of the family Amaryllidaceae and its bearing on the interpretation of its phylogeny. Genet. Iber. 6: $71-100$.

- and Majumdar, Arati 1955. Cytological peculiarity of Pteris longifolia and its importance in evolution. Sci. Cuit. 21: 338-339.

- and Sarkat, S.K. 1956. Cytology of different species of Palms and its bearing on the solution of the problems of phylogeny and speciation. Genetica 28: 361-488.

- and Sharma, Archana (nee Mookerjea) 1956. Fixity in chromosome number of plants. Nature 177: 335-336.

- and - 1957. Karyotype studies in Cestrum as an aid to the identification of species. Genetica 29: 83-100.

Stewart, R.N. 1948. The morphology of the somatic chromosomes of Lilium. Amer. J. Bot. 34: 9-26. 